\title{
AN INTELLIGENT PATIENT TELE-MONITORING SYSTEM USING ANDROID TECHNOLOGY
}

\author{
Anbumani.S ${ }^{1}$, Kavin. $\mathbf{R}^{2}$, Saranya.A ${ }^{3}$, Dineshkumar. $T^{4}$, Premkumar. $^{5}$ \\ ${ }^{1}$ Assistant Professor, ECE Department, Excel Engineering College, Tamil Nadu, India \\ ${ }^{2}$ Assistant Professor, EEE Department, Excel College of Engineering \& Technology, Tamil Nadu, India \\ ${ }^{3}$ Saranya.A, Final Year Student ECE Department, Excel Engineering College, Tamil Nadu, India \\ ${ }^{4}$ Dineshkumar.T, Final Year Student ECE Department, Excel Engineering College, Tamil Nadu, India \\ ${ }^{5}$ Premkumar.R, Final Year Student ECE Department, Excel Engineering College, Tamil Nadu, India
}

\begin{abstract}
Healthcare to people anytime and anywhere in the world in a more economic and patient friendly manner. Therefore for increasing the patient care efficiency, there arises a need to improve the patient monitoring devices and make them more mobile. The medical world today faces two basic problems when it comes to patient monitoring. Firstly, the needs of health care's provider's presence near the bedside of the patient and secondly, the patient is restricted to bed and wired to large machines. In order to achieve better quality patient care, the above cited problems have to be solved.As telecommunications technologies are advancing, it has become feasible to design more portal vital sign patient tele monitoring systems to acquire, record, display and to transmit the physiological signal from the patient to any place.In this project real time transmission of patient self -tested blood pressure data to doctor is achieved.This system is very convenient to use for doctors and patient. The modern visionary of healthcare industry is to provide better
\end{abstract}

Keywords: GSM, Android

\section{INTRODUCTION}

\subsection{System Overview}

Heart failure and stroke cause a big burden on society due to their high costs of care, lower quality of life and premature death. It causes million of death worldwide [1].

The monitoring of patients physiological information are very important for the further treatment. Many patients can benefit from continuous monitoring as a part of a diagnostic procedure, optimal maintenance of a chronic condition or during supervised recovery from an acute event or surgical procedure. The remote monitoring also enables the patient to live his normal live and help decreasing the cost of healthcare. With the recent advance in IC design, the computing power and the memory size of mobile device have increased considerably. This development makes many mobile devices, capable of carrying out complex computing tasks and thus can be used in monitoring heart disease patient remotely

In this paper a real-time GSM system that facilitates the monitoring and follows up of the patient's condition is implemented. The electrocardiogram (GSM) is a test that records the electrical activity of the heart. GSM translates the heart's electrical activity into line tracings on paper. These records hold significant information that can be used to measure how fast the heart is beating and to detect the irregularity of the heartbeats. The computation power of mobile devices is utilized in this system. The proposed system has good extensibility and can easily incorporate other physiological signals to suit various tele-health scenarios. The system has two parts one carried by users, e.g. chronic patient, and the second carried by service providers e.g., the medical doctors.

\subsection{Related Works}

The use of telecommunications for remote diagnosis is growing rapidly, and there are several products and projects within mobile GSM recording using Internet solutions [2], Bluetooth technology [3], cellular phones [4], [5], WAPbased implementations [6] and wireless local area networks, WLANand Wireless Sensor Networks [7], [8], [9].

A remote diagnosis system integrating digital telemetry has been developed, using a wireless patient module, a homecare station and a remote clinical station [10]. Some ECG-recording systems, like "Holter-monitoring", are using built-in mobile telephones tosend information to the hospital [11], but are mostly used with a recording unit that physicallyhas to be carried to the hospital for analysis. In [12], [13] a wireless and wearable electrocardiogram (ECG) sensor transmitting signals to a diagnostic station at the hospital is introduced. An ECG system has been proposed based on mobile platform which transmits abnormal heartbeats identified in a patient-worn unit [14]. Another ECG analyser system has been developed to capture, record, and analyze ECG signals on a PDA device carried by the patient [15]. In [16], a Decision Support System (DSS) prototype has been provided to deliver an ECG signal to a hand-held device which will be capable of providing remote mobile communication to speed up diagnosis. 
In the electrical and computer engineering department of Cornell University, an ECG hardware design has been implemented to measure the electrical activity of the heart [17].

Another paper presented the design, implementation, and results related to the storage system of medical information associated to the ECG signal [18].

The objective of this research is:

a. Design an affordable and cheap GSM system as compared with the available GSM systems.

b. Design afully online system that monitors the patient status in real time and sends the results to health care server.

c. Add features to the current available system such as:

i. Detecting abnormalities and send alert message immediately to doctors via e-mail and SMS

ii. Develop Android based application that helps doctors to follow up their patients remotely via Web page, email, and smart phone.

\subsection{Paper Organization}

The rest of the paper is organized as follows. In section two the system architecture is given. Section 3 presents patient subsystem. In Section 4, Web Server and Database Subsystem are described. Section 5 discusses the Android unit subsystem.

Section 6 shows the results and discussion. The last section is a discussion on future work and conclusion.

\section{SYSTEM ARCHITECTURE}

As shown in figure (1) the system consists of the following subsystems:

1. Patient unit subsystem:This includes electrodes that sense the electrical activity going through the heart, signal amplification circuit, conditioning circuit, data acquisition circuit and home gateway. The circuit takes a reading every 30 minutes and sends it to home gateway $\mathrm{PC}$.

2. Web Server and Database subsystem: To store the patient GSM signal data, detect any abnormality in the GSM signal and publish the results that can be accessed only by authorized people.

3. Android unit subsystem: Android based application that enables doctors to access the patient details using smart phone

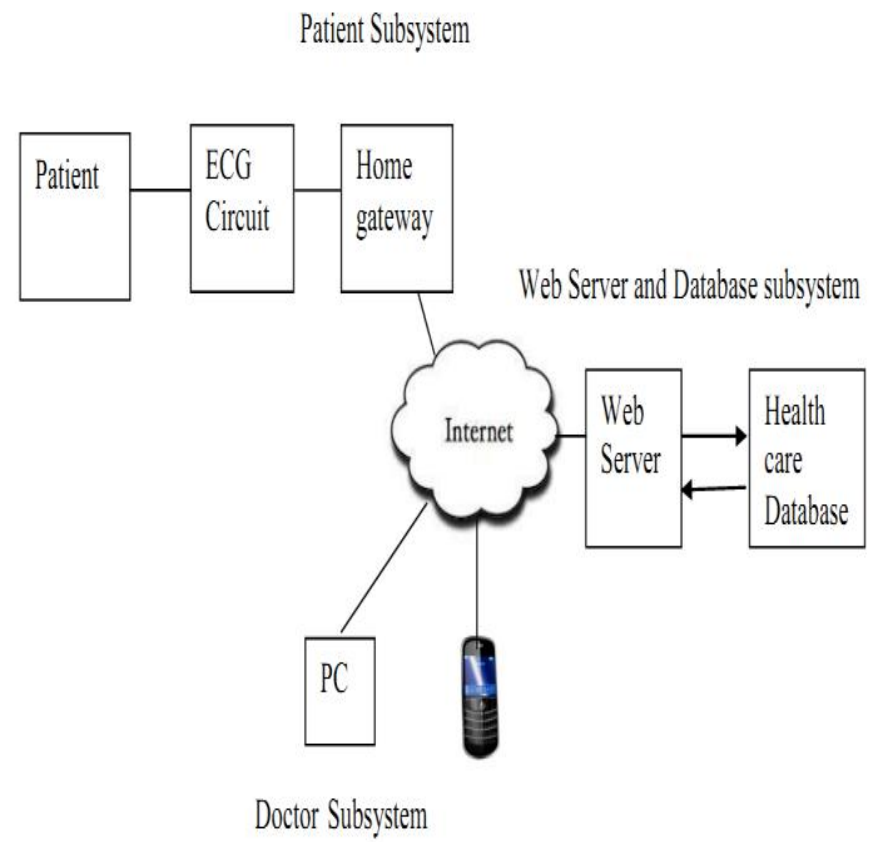

\section{PATIENT UNIT SUBSYSTEM}

The patient subsystem shown in figure (2) contains the following components:

- $\quad$ Electrodes and cables (for the right arm, leftarm, and right leg).

- $\quad$ Right leg drive for the ground reference.

- Instrumentation Amplifier (IA).

- Signal conditioning (low pass filter, band pass filter, and notch filter).

- Data logger and Acquisition System (DAQ).

- Home gateway

The GSM signals have Electrode contact noise such as loose contacts, motion artifacts, and baseline drift due to respiration [19]. They also pick electro-magnetic interference caused by other electronic devices surrounding the GSMdevice and electrodes amplitude of $1 \mathrm{mV}$ only, we need to filter the signal with a Low Pass, Band Pass, and a Notch filters. Finally, the resulted signal must be amplified. Next the ECG signals need to be exported to the home gateway. In this project, USB DrDAQ data logger is acquiring the ECG data. It connects to the PC on a USB 2.0 port and to the ECG circuit's output using a probe through the scope channel of the DAQ.

The home gateway could be any PC, laptop, iPad, PDA or any other device that can be connected to the Internet. The Home gateway will receive the ECG signal from the data logger and sends it to the healthcare server. 


\section{WEB SERVER}

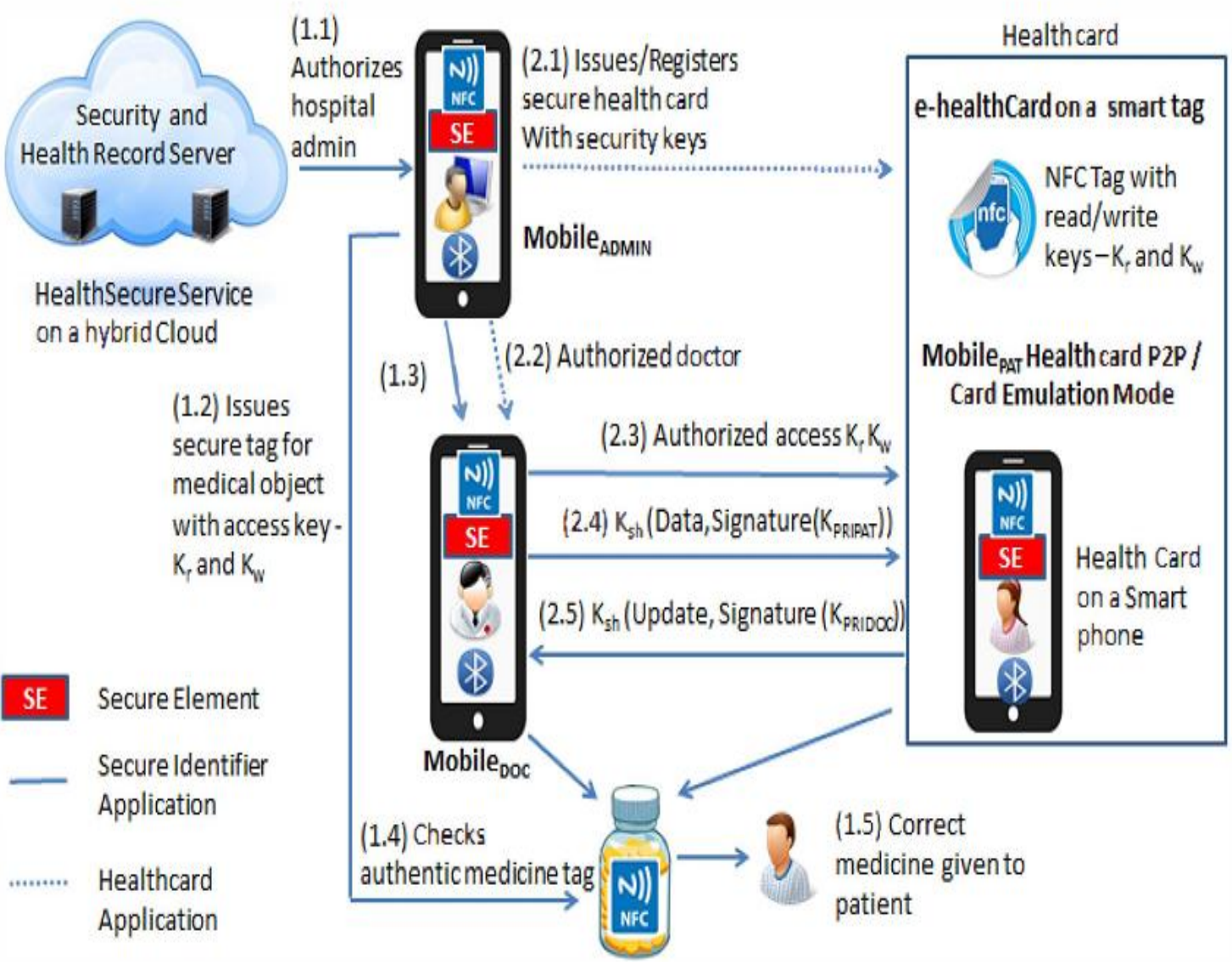

\subsection{Web Server Implementation}

The designed ASP.NET website gives the doctors the ability to monitor their patients' condition remotely.

The website has three types of users:

- Web Admin: responsible for creating new accounts for both patients and doctors, manage their profiles, and the process of assigning doctors to patients.

- Doctor: Can access his/her assigned patients' ECG readings, add and view comments on his patients, add and view medical reports of his patient written by him/her or another doctor in charge of this patient.

- Patient: Can access his/her medical reports and view the doctor / doctors assigned to him/her.

The doctor can "Filter" or search the patients either by name, File number, ID, or ID type. In order to get the full list he / she just can click the "Clear" button. The "select" column is used to access the information of a specific patient

\section{ANDROID UNIT SUBSYSTEM}

Two android applications were developed, one for the patient and another for the doctor, each having different functionality to suit the user.

\subsection{Doctor Application (GSM Note):}

Doctor home page contains a list of the patient's names. The status of each patient can be seen within each patient name, to indicate whether he / she are online or not. The Green light or the Red will be shown to indicate whether the patients are in danger or not. This is useful for the emergency cases, so that the doctor can communicate directly with the patient. The doctor can access any patient info by pressing on his/her photo to see his/her details, condition and other important info such his hear beat rate. 


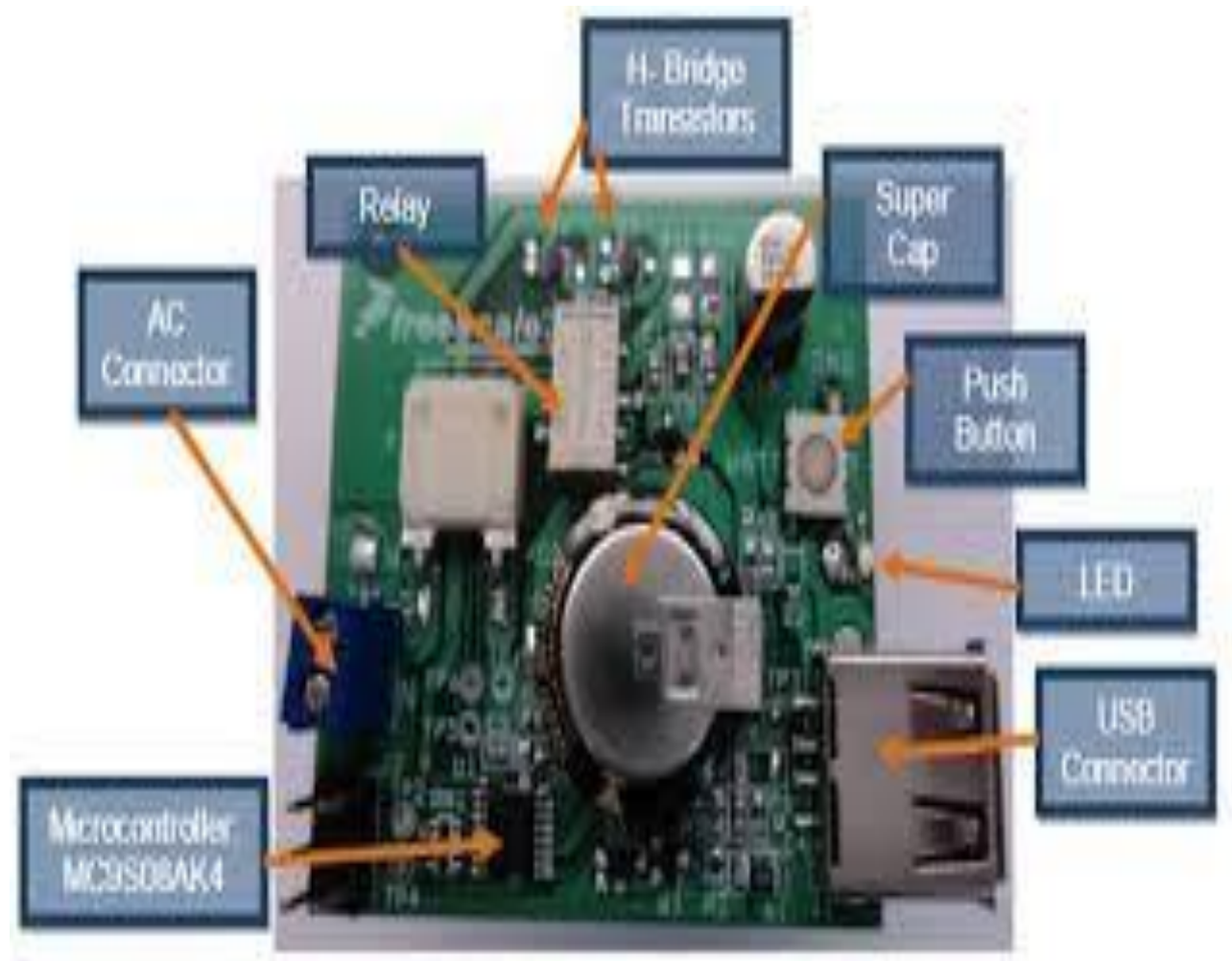

\subsection{Patient Application (My Note):}

The patient home page contains a list of icons. These icons are "Your condition", "schedule", "share", and "options".

The status of the patient can be seen at the top of the page, which is displayed in a Green or Red square.

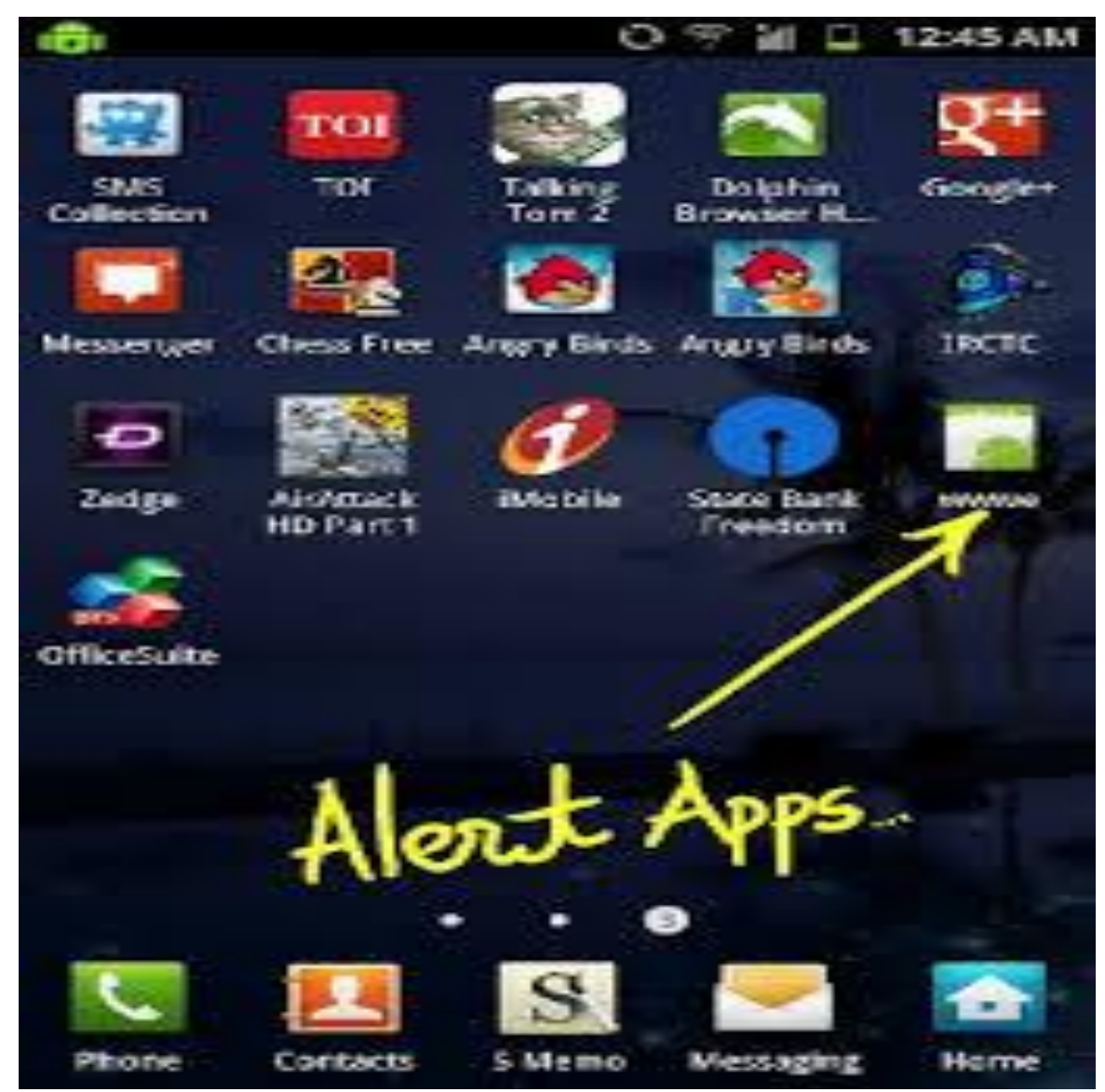




\section{RESULTS AND DISCUSSIONS}

The system have been implemented, tested and achieved the design objectives mentioned in section 1.3 which are the design of affordable and cheap ECG system as compared with the available ECG systems as well as the streaming of the patient ECG to the health care provider web site so that doctors can monitors their patient status in real time. Added features like detecting the abnormalities in patient ECG and send alert message immediately to doctors via e-mail and SMS is also implemented and tested. FinallyAndroid based application that helps doctors to follow up their patients remotely via smart phone is implemented and tested.

\section{CONCLUSION}

In this paper a real-time low cost heart disease monitoring system is introduced. The developed system produces a live GSM system that shows the real-time electrical activity of the heart, its rate, and analyze the readings for five of the most common and vital abnormalities

If the system detects any of these abnormalities it will alert the doctor and hospital by sending email and SMS message. The system also implements an application based on Android platform for doctors and for patients. The doctor application provide online information about the patient status such as the patient's heart beat rate, ECG, patient history and provides new reading every 30 minutes. The system enables doctors to remotely follow-up the status of their patient using their smart phones. The system was tested and checked by medical teams for validation and the system ECG generation and abnormality detection were certified. This system provides some sort of freedom to both doctor and patient since the results are shown at real-time on a website and the doctor will be alerted on his/her Android device in case of abnormality detection. Also, it can be deployed as part of a Decision Support System (DSS) in hospitals.

The cost of the components from which the system was assembled is equal to USD50. As a future work, the system can be enhanced more using Programmable system on chip integrated with Biomedical GSM sensor to decrease the system size and get better response time.

\section{REFERENCES}

[1]. R. Roberts, "Use of Remote Monitoring Devices Increases, Telemedicine Information Exchange", Wall Street Journal, April 18, 2006.

[2]. R. Fensli E. Gunnarson, and O. Hejlesen, "A Wireless Cardiac Alarm System for Continuous Event Recording," Medinfo2004, San Francisco, USA, pp 1598,2004.

[3]. A. Thakor, R. Kher and D. Patel, "Wearable ECG Recording and Monitoring System based on MSP430 Microcontroller", International Journal of Computer Science and Telecommunications, Volume 3, Issue 10, pp. 40-43, October 2012

[4]. Q. Fang, F. Sufi and I. Cosic, " A Mobile Device Based ECG Analysis System", Data Mining in Medical and
Biological Research, Book edited by: Eugenia G. Giannopoulou, ISBN 978-953-7619-30-5, pp. 320, December 2008, I-Tech, Vienna, Austria.

[5]. S. Gradl, P. Kugler, C. Lohmuller, B. Eskofier, "Realtime ECG monitoring and arrhythmia detection using Android-based mobile devices", Conf Proc IEEE Eng Med Biol Soc., pp. 2452-2455, 2012.

[6]. K. Hung , Y. Zhang Y, "Implementation of a WAPbased telemedicine system for patient monitoring", IEEE Trans Information Technology in Biomedicine, pp 101-107, Vol. 2 issue 7, Jun 2003.

[7]. P. Pandian, K. Safeer, P. Gupta, D. Shakunthala, B. Sundersheshu and V. Padaki, "Wireless sensor network for wearable physiological monitoring" journal of networks, Vol. 3, no. 5, pp. 21-29, may, 2008.

[8]. J. Lin, Y. Chang, C. Liu and K. Pan, Wireless Sensor Networks and Their Applications to the Healthcare and Precision Agriculture, Wireless Sensor Networks, (Ed.), ISBN: 978-953-307-325-5, INTECH Publisher, 2011.

[9]. M. Aminian and H. Reza Naji ," A Hospital Healthcare Monitoring System Using Wireless Sensor Networks", journal of Health \& Medical Informatics, Vol4. Issue 2, pp. $1-6,2013$.

[10]. Y. Nam, Z. Halm, Y. Chee, and K. Park, "Development of remote diagnosis system Integrating digital telemetry for medicine," presented at

Proceedings of the 20th Annual International Conference of the IEEE Engineering in Medicine and Biology Society, pp. 1170-1173, 1998.

[11]. Schiller, "ECG HOLTER Recorder MT-120."[Online] http://www.schiller.ch/products, 2004.

[12]. R. Fensli, E. Gunnarson, T. Gundersen, "A Wearable ECG-recording System for Continuous Arrhythmia Monitoring in a Wireless Tele-Home-Care Situation", 18 IEEE International Symposium on Computer based Medical System, Dublin June 23-24, 2005.

[13]. R. Fensli, E. Gunnarson, and O. Hejlesen, “A Wireless ECG System for Continuous Event Recording and Communication to a Clinical Alarm Station," 26th Annual International Conference of the IEEE Engineering in Medicine and Biology Society, San Francisco, USA; pp 2208-11, 2004

[14]. W. Cheng, Y. Ming-Feng., C. Kuang-Chiung, L. RenGuey, "Real-time ECG telemonitoring system with mobile phone platform", Measurement, Volume 41, Issue 4, pp. 463-470, 2008.

[15]. J. Rodriguez, L. Dranca, A. Goñi, A. Illarramendi, "Web access to data in a mobile ECG monitoring system", Studies in Health Technology and Informatics, 105: pp. 100111, 2004.

[16]. L. Meng-Kuan, et. al, "Development of a prototype multi-touch ECG diagnostic decision support system using mobile technology for monitoring cardiac patients at a distance". In: 15th Pacific Asia Conference on Information Systems, 2011, Brisbane, Australia.

[17]. Cornell University, EKG Monitoring System, [Online] Available at: http://people.ece.cornell.edu/land/courses/ece4760/FinalProj ects/s2001/j1175/EE476.htm 
[18]. A. Rubiano, et al, "Design of Medical Storage SystemECG Signal", World Academy of Science, Engineering and Technology 59, 2011.

[19]. University of South California, "Noise in ECG and How to Deal With it", [Online] Available at:

http://wwwclasses.usc.edu/engr/bme/620/LectureECGNoise. pdf

[20]. Physionet site, [Online] Available at: http://www.physionet.org/

[21]. S. Das, M. Chakraborty, "Comparison of Power Spectral Density (PSD) of Normal and Abnormal ECG", IJCA Special Issue on "2nd National ConferenceComputing, Communication and Sensor Network" CCSN issue 4,pp. 1-14, 2011

[22]. H. Wan, J. Cammarota, A. Akin, H. Sun, "Comparison of QRS peak detection algorithms in extracting HRV signal", in Proceedings - 19th International Conference IEEE/EMBS, Oct. 30 - Nov. 2, 1997.

[23]. R. Sheikh, I.Taj, "Cardiac disorder diagnosis based on ECG segments analysis and classification" in IEEE 978-14244-4361-1, 2009.

[24]. J. Pan, W.J. Tompkins, "A real-time QRS detection algorithm”, IEEE Trans. Biomed. Eng., vol.32, pp. 230-236, 1985.

[25]. W.J. Tompkins, Biomedical Digital Signal Processing: C Language Examples and Laboratory Experiments For The IBM PC, Prentice Hall, 2000.

\section{BIOGRAPHIES}

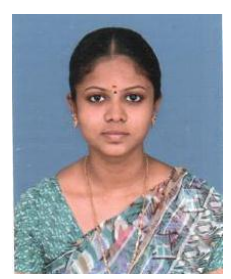

S. Anbumani was born in the year of 1989, at Bhavani. She did B.E (BME) at Vellalar College of Engineering \& technology in the year of 2010. She did her M.E (Applied Electronics) at SSM college of Engineering in the year of 2012. Currently she is working as Assistant Professor in the Dept. of ECE at Excel Engineering College, Komarapalayam, Namakkal District, Tamilnadu, India.

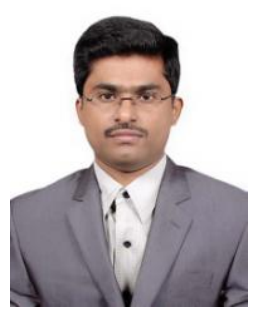

R. Kavin was born in the year of 1985, at komarapalayam. He did B.E (EEE) at KSR College of technology in the year of 2007. He did his M.E (PED) at Sri Venkateswara college of Engineering in the year of 2010, Sriperumpudhur, Chennai, Tamilnadu, India, Currently he is working as Assistant Professor in the Dept. of EEE at Excel College of Engineering \& Technology, Komarapalayam, Namakkal District, Tamilnadu, India. 